\title{
TRAIL-producing NK cells contribute to liver injury and related fibrogenesis in the context of GNMT deficiency
}

\author{
Sara Fernández-Álvarez ${ }^{1}$, Virginia Gutiérrez-de Juan ${ }^{1}$ Imanol Zubiete-Franco ${ }^{1}$, Lucia Barbier-Torres ${ }^{1}$, \\ Agustín Lahoz ${ }^{2}$, Albert Parés ${ }^{3}$, Zigmund Luka ${ }^{4}$, Conrad Wagner ${ }^{4}$, Shelly C Lư ${ }^{5}$, José M Mato ${ }^{1}$, \\ María L Martínez-Chantar ${ }^{1,6}$ and Naiara Beraza, ${ }^{1,7}$
}

Glycine-N-methyltransferase (GNMT) is essential to preserve liver homeostasis. Cirrhotic patients show low expression of GNMT that is absent in hepatocellular carcinoma (HCC) samples. Accordingly, GNMT deficiency in mice leads to steatohepatitis, fibrosis, cirrhosis, and HCC. Lack of GNMT triggers NK cell activation in GNMT ${ }^{-/}$mice and depletion of TRAIL significantly attenuates acute liver injury and inflammation in these animals. Chronic inflammation leads to fibrogenesis, further contributing to the progression of chronic liver injury regardless of the etiology. The aim of our study is to elucidate the implication of TRAIL-producing NK cells in the progression of chronic liver injury and fibrogenesis. For this we generated double $\mathrm{TRAIL}^{-/-} / \mathrm{GNMT}^{-/-}$mice in which we found that TRAIL deficiency efficiently protected the liver against chronic liver injury and fibrogenesis in the context of GNMT deficiency. Next, to better delineate the implication of TRAIL-producing NK cells during fibrogenesis we performed bile duct ligation (BDL) to GNMT ${ }^{-1-}$ and $\mathrm{TRAIL}^{-/-} / \mathrm{GNMT}^{-1-}$ mice. In $\mathrm{GNMT}^{-/-}$mice, exacerbated fibrogenic response after BDL concurred with NK1.1 ${ }^{+}$cell activation. Importantly, specific inhibition of TRAIL-producing NK cells efficiently protected $\mathrm{GNMT}^{-1-}$ mice from $\mathrm{BDL}$-induced liver injury and fibrogenesis. Finally, $\mathrm{TRAIL}^{-/-} / \mathrm{GNMT}^{-1-}$ mice showed significantly less fibrosis after BDL than $\mathrm{GNMT}^{-1-}$ mice further underlining the relevance of the TRAIL/DR5 axis in mediating liver injury and fibrogenesis in $\mathrm{GNMT}^{-1-}$ mice. Finally, in vivo silencing of DR5 efficiently protected $\mathrm{GNMT}^{-1-}$ mice from BDL-liver injury and fibrogenesis, overall underscoring the key role of the TRAIL/DR5 axis in promoting fibrogenesis in the context of absence of GNMT. Overall, our work demonstrates that TRAIL-producing NK cells actively contribute to liver injury and further fibrogenesis in the pathological context of GNMT deficiency, a molecular scenario characteristic of chronic human liver disease.

Laboratory Investigation (2015) 95, 223-236; doi:10.1038/labinvest.2014.151; published online 22 December 2014

Glycine-N-methyltransferase (GNMT) is the most abundant methyltransferase in the liver. The relevance of GNMT in preserving liver homeostasis relies on its ability to tightly control the catabolism of SAMe, the main methyl donor of the body. ${ }^{1}$ GNMT is downregulated in cirrhotic patients (from HCV and ASH etiologies) and is absent in HCC samples. ${ }^{2}$ In accordance, we described that mice lacking GNMT $\left(\mathrm{GNMT}^{-1-}\right)$ develop spontaneous steatosis that progresses to steatohepatitis, cirrhosis, and HCC. ${ }^{3}$ More recently, we found that GNMT deficiency correlates with strong activation of NK cells, which mediate endotoxin-mediated inflammation and acute liver injury through TRAIL. ${ }^{4}$ Moreover, we found that GNMT-deficient livers and hepatocytes expressed more TRAILR2/DR5, further suggesting that the TRAIL/DR5 axis may have a key role in the progression of NASH that spontaneously develop in $\mathrm{GNMT}^{-1-}$ animals. Nevertheless, the implication of TRAIL/NK cells during chronic liver injury and fibrogenesis was not further explored.

Chronic liver injury leads to fibrogenesis and eventually to cirrhosis and HCC. Fibrosis is a common feature of the

\footnotetext{
${ }^{1}$ Department of Metabolomics, CIC bioGUNE, Centro de Investigación Biomédica en Red de Enfermedades Hepáticas y Digestivas (Ciberehd), Derio, Spain; ${ }^{2}$ Unidad de Hepatología, Instituto de Investigación Sanitaria La Fé, Valencia, Spain; ${ }^{3}$ Liver Unit, Hospital Clínic, IDIBAPS, CIBERehd, University of Barcelona, Barcelona, Spain;

${ }^{4}$ Department of Biochemistry, Vanderbilt University School of Medicine, Nashville, TN, USA; ${ }^{5}$ Division of Gastrointestinal and Liver Diseases, USC Research Center for Liver Diseases, Southern California Research Center for Alcoholic Liver and Pancreatic Diseases and Cirrhosis, Keck School of Medicine, University of Southern California, Los Angeles, CA, USA; ${ }^{6}$ Department of Biochemistry and Molecular Biology, University of the Basque Country (UPV/EHU), Bilbao, Spain and ${ }^{7}$ Ikerbasque, Basque Foundation for Science, Bilbao, Spain

Correspondence: Dr N Beraza, PhD, Department of Metabolomics, CIC bioGUNE, Centro de Investigación Biomédica en Red de Enfermedades Hepáticas y Digestivas (Ciberehd), Technology Park of Bizkaia, 48160 Derio, Bizkaia, Spain.
}

E-mail: nberaza@cicbiogune.es

Received 3 July 2014; revised 16 October 2014; accepted 27 October 2014 
pathogenesis of chronic liver disease regardless of the etiology; NASH, HCV infection, alcohol abuse, primary biliary cirrhosis (PBC), and autoimmune hepatitis. ${ }^{5}$ In the context of chronic liver injury, inflammation actively contributes to fibrogenesis, although the molecular mechanisms underlying this progression are poorly understood. It is commonly accepted that hepatocyte apoptotic cell death promotes an inflammatory response to remove cell debris, which in turn activates hepatic stellate cells (HSCs) to deposit collagen, in a tissue remodeling/scarring process. Kupffer cells (KCs) are the main cell compartment mediating this process, although HSC can also be directly activated by phagocytosis of apoptotic hepatocytes. ${ }^{6,7}$ Thus, the innate immune system and HSC are closely linked during fibrogenesis.

NKT/NK cells are part of the innate immune system, representing the first line of defense of the liver. NKT and NK cells seem to have differential roles during fibrogenesis. Thus, increased presence of NKT cells in cirrhotic livers contributes to fibrogenesis during $\mathrm{NASH},{ }^{8}$ whereas NK cells are commonly described as antifibrogenic due to their ability to promote apoptosis of HSC through TRAIL/DR5 and NKG2D-RAE1. ${ }^{9,10}$ Interestingly, a number of reports show NK cell activation during cholestatic liver diseases in patients. Thus, NK cells have a cytotoxic effect against autologous biliary cells/cholangiocytes in PBC and PSC patients ${ }^{11-13}$ and TRAIL produced by NK cells contributes to cholestatic liver injury in mice. ${ }^{14}$ In addition, in the context of NASH progression the presence of major histocompatibility complex A and $\mathrm{B}$ proteins (MIC $\mathrm{A} / \mathrm{B})$, stress ligands recognized by NK cells, directly correlate with the fibrosis stage in patients. ${ }^{15}$ Overall, these studies suggest the potential implication of NK cells in mediating liver injury during chronic liver disease, although its fibrogenic role remains uncertain.

Taking all this together, in the present work we aim to investigate the molecular mechanisms leading to fibrogenesis in the pathological context of GNMT deficiency, a scenario found in cirrhotic patients. Here, we provide evidence that TRAIL is a key factor in the development of the spontaneous pathogenic phenotype observed in the liver of GNMT-deficient animals. In addition, we describe that NK cell activation concurs with exacerbated fibrogenesis in the context of GNMT deficiency after bile duct ligation (BDL). Finally, through different experimental strategies we show that selective inhibition of TRAIL-producing NK cells, knockdown of TRAIL and in vivo silencing of DR5 in $\mathrm{GNMT}^{-1-}$ mice efficiently protects the liver from BDL-induced liver injury, overall attenuating fibrogenesis in $\mathrm{GNMT}^{-1-}$ mice.

\section{MATERIALS AND METHODS GNMT Knockout Animals}

$\mathrm{GNMT}^{-1-}$ mice were generated as previously described. ${ }^{16}$ TRAIL $^{-1-}$ mice were kindly provided by Amgen. Double KO mice were obtained crossing $\mathrm{GNMT}^{-1-}$ with $\mathrm{TRAIL}^{-1-}$ mice. Male 8- to 12-week-old mice were used in our experiments. BDL was performed as previously described. ${ }^{17}$
Animals were treated according to the guidelines of the National Academy of Sciences (National Institutes of Health publication 86-23, revised 1985). Animal husbandry and procedures were approved by the Department of Environment, Planning, Agriculture and Fisheries of the Basque Country Government. All experiments shown in this MS were performed following the guidelines of the CICbioGUNE Facility with AALAC certificate.

\section{Immunohistochemistry}

Paraffin-embedded liver samples were sectioned, dewaxed, and hydrated. Immunohistochemistry (IHC) was performed using GNMT Ab, F4/80 (Serotec) and nuclei were counterstained with hematoxylin. Fibrosis was determined with Sirius Red staining and IF using $\alpha$ Smooth Muscle Actin ( $\alpha$ SMA) $\mathrm{Ab}$ (Sigma), labeled with Cy3 (red). Nuclei were counterstained with DAPI (blue). Quantification of F4/80, $\alpha$ SMA, and collagen deposition in Sirius Red-stained sections were analyzed using the Frida software and represented as the \% of the stained area relative to the total area (power field). Five to ten fields per sample were pictured and analyzed.

\section{Liver Injury Experimental Model}

BDL was performed as previously described. ${ }^{17}$

\section{Human PBC Samples}

GNMT was assessed using IHC in sections of deparaffinized liver samples obtained by percutaneous biopsy in five patients and samples from the explanted liver in three patients. The diagnosis of $\mathrm{PBC}$ was established by liver biopsy with characteristic features of the disease and presence of antimitochondrial antibodies. Biochemical and histological cholestasis were very prominent in the explanted samples, whereas features of chronic nonsuppurative cholangitis, portal inflammation, and ductopenia with fewer prominent biochemical cholestasis were present in the five patients in the initial stages of the disease. All the latter patients had Ludwig's stage I, whereas two of the explanted livers had cirrhosis and the remaining livers had septal fibrosis (Ludwig's stage III) and severe ductopenia. The Ethical Committee of the institution approved the study and all participants provided written informed consent.

\section{Characterization of Liver Damage and Cell Death}

Alanine aminotransferase (ALT), aspartate aminotransferase (AST), and bilirubin were determined in serum samples. Histological analysis of the liver parenchyma was performed in liver sections that were stained with hematoxylin \& eosin (H\&E). Cell death was determined on frozen liver sections with TUNEL assay using the In Situ Cell Death Detection Kit (Roche diagnosis) following the manufacturer's instructions. Caspase-3 activity was quantified in snap-frozen livers as described. ${ }^{18}$ The presence of TNF was determined by ELISA (R\&D systems) in whole-liver extracts. 


\section{RNA Isolation and Quantitative Real-Time PCR}

RNA was isolated with TriZol Reagent (Invitrogen) followed by first-strand synthesis with Oligo dT primers and reverse transcription with M-MLV Reverse Transcriptase (Invitrogen). Quantitative real-time PCR (RT-PCR) was performed using SYBR Green reagent (Quantas biosciences) in a MyiQ single color Real-time PCR detection system (Bio-Rad). Gene expression was normalized using glyceraldehyde 3-phosphate dehydrogenase (GAPDH) and expressed in times vs control expression. Primers can be provided under request.

\section{Western Blot Analysis}

Whole-liver protein extracts were resolved in sodium dodecyl sulfate-polyacrylamide gels and transferred to nitrocellulose membranes (Whatman). Membranes were probed with the following primary antibodies: DR5 and p-JNK1/2 (Thr 183/ Tyr185) from Cell signaling. FADD was obtained from Santa Cruz Biotechnology. As a loading control, we used GAPDH antibody (Abcam). As secondary antibodies, we used antirabbit-IgG-HRP-linked (Cell Signaling) and anti-mouse IgG-HRP-linked (Santa Cruz Biotechnology).

\section{MNC Isolation and Flow Cytometric Analysis}

Liver MNCs were obtained as previously described. ${ }^{19}$ In brief, liver MNCs were isolated by enzymatic digestion with collagenase IV (Worthtington) during $30 \mathrm{~min}$ at $37^{\circ}$. Extracts were passed through a $12-\mu \mathrm{m}$-mesh (Becton Dickinson). MNCs were pelleted and washed by centrifugation at $300 \mathrm{~g}$ for $10 \mathrm{~min}$. Erythrocytes were removed using PharmLyse lysing buffer (BD). Cells were stained with CD45-APC-Cy7, CD3-APC, NK1.1-PE-Cy7, and TRAIL-PE (BD). Flow cytometry analysis was performed on a FACS Canto II (BD) and data were analyzed with the FlowJo software (Tree Star). $\mathrm{NK} 1.1^{+} / \mathrm{CD} 3{ }^{-}(\mathrm{NK})$ and $\mathrm{NK} 1.1^{+} / \mathrm{CD}^{+}{ }^{+}(\mathrm{NKT})$ cells were isolated in a FACSaria cell sorter.

\section{NK/NKT and NK Cell Depletion}

NK/NKT and specific NK cell depletion was achieved by i.p. administration of $250 \mu \mathrm{g} /$ mouse of $\mathrm{NK}_{1.1}{ }^{+}$antibody purified from hybridoma PK-136 (American Type Culture Collection) culture supernatants as previously described ${ }^{19}$ and by ASIALO, respectively. Both ASIALO and NK1.1 ${ }^{+}$Abs were injected $24 \mathrm{~h}$ before BDL and every $72 \mathrm{~h}$ after surgery. As control group, $\mathrm{GNMT}^{-1-}$ mice were injected with IgG $(250 \mu \mathrm{g} /$ mouse from BD Bioscience) at the same time points as ASIALO and NK1.1 ${ }^{+} \mathrm{Ab}$.

\section{In vivo DR5 Overexpression}

The TRAILR2/DR5 short hairpin (sh)RNA was cloned into the retroviral expression vector PSM2C (Open Biosystems) to induce sequence-specific silencing of DR5 in $\mathrm{GNMT}^{-1-}$ mice. ShDR5 was administered by hydrodynamic i.v. injection at $24 \mathrm{~h}$ before BDL and every $72 \mathrm{~h}$ after surgery.

\section{Statistical Analysis}

Data are expressed as mean \pm s.d. of the mean. Statistical significance was determined by two-way analysis of variance followed by a Student's $t$-test.

\section{RESULTS \\ Reduced GNMT Expression in Patients with Cholestatic Liver Disease}

Previous work showed that GNMT expression is significantly downregulated in cirrhotic patients from alcohol and HCV etiologies. ${ }^{2}$ Here we show that expression of GNMT was also decreased in the context of chronic cholestatic liver disease. Thus, livers from patients with PBC, both at initial stages and in patients with advanced disease who were transplanted, had significantly lower levels of GNMT than healthy livers as shown by IHC and further quantification (Figures $1 \mathrm{a}$ and $\mathrm{b}$ ). The finding that attenuation of GNMT was found at early stages of cholestatic liver disease, where fibrosis is not prominent, points to GNMT deficiency as a contributor/ mediator of fibrogenesis rather than a consequence.

\section{TRAIL Deficiency Reverts the Spontaneous Damaging Phenotype found in GNMT ${ }^{-1-}$ Mice}

Supporting the relevance of GNMT as a key factor in preserving liver homeostasis, we previously described that GNMT-deficient mice $\left(\mathrm{GNMT}^{-/-}\right)$show spontaneous steatohepatitis, cirrhosis, and HCC development, mimicking the pathogenesis of NASH in humans. ${ }^{3}$ Importantly, GNMT deficiency correlated with NK cell activation already at initial stages of liver disease, when mice show only a mild damaging phenotype. ${ }^{4}$ Moreover, TRAIL-producing NK cell depletion efficiently protected the liver against endotoxin-induced liver injury. However, the implication of TRAIL in the context of chronic liver disease was not further examined.

In order to investigate the contribution of TRAIL to chronic liver disease we generated TRAIL- and GNMT-deficient mice (TRAIL $^{-l-} / \mathrm{GNMT}^{-1-}$ ). TRAIL $^{-l-} / \mathrm{GNMT}^{-1-}$ mice showed an apparent remission of the spontaneous damaging phenotype found in $\mathrm{GNMT}^{-1-}$ mice. ${ }^{3} \mathrm{H} \& \mathrm{E}$ staining evidenced a clear attenuation of the liver parenchymal damage, especially in 9-month-old TRAIL ${ }^{-/-} / \mathrm{GNMT}^{-1-}$ mice compared with $\mathrm{GNMT}^{-1-}$ animals (Figure 2a). Moreover, we observed a significant reduction of liver fibrogenesis in 3- and 9-month-old TRAIL ${ }^{-1-} / \mathrm{GNMT}^{-1-}$ mice compared with $\mathrm{GNMT}^{-1-}$ animals as evidenced by Sirius red staining (Figure 2b) and further quantification using the Frida software (Figure 2c). Further analysis using qRT-PCR confirmed the beneficial impact of TRAIL deficiency in the spontaneous fibrogenesis observed in $\mathrm{GNMT}^{-1-}$ animals as evidenced by lower expression of collagen 1A1, $\alpha \mathrm{SMA}$, and TGF $\beta$ found in 9-month-old $\mathrm{TRAIL}^{-/-}$/ GNMT $^{-1-}$ mice (Figure 2d). Macroscopical analysis further confirmed the important beneficial impact of TRAIL deficiency on GNMT-deficient mice in the attenuation of the cirrhotic/tumorigenic phenotype found in $\mathrm{GNMT}^{-/-}$mice 



Figure 1 Low GNMT expression in human PBC livers. (a) IHC and (b) further quantification using the FRIDA software showing lower GNMT expression in human samples from PBC patients at initial stages or after transplantation compared with healthy controls. $n=8-10$. ${ }^{* *} P<0.01$ (healthy vs early PBC and healthy vs late PBC). Error bars represent s.d.

at 9 months of age (Figure 2e). Importantly, the improved phenotype found in TRAIL ${ }^{-1-} / \mathrm{GNMT}^{-1-}$ mice correlated with a significantly lower activation of NK cells. Hence, we found a significantly higher number of NK1.1 ${ }^{+} / \mathrm{CD}^{-}$(NK) cells in TRAIL $^{-1-} / \mathrm{GNMT}^{-1-}$ animals compared with $\mathrm{GNMT}^{-1-}$ mice at 3 months of age (Figure 2f). Restoration of the presence of NK cells to a percentage comparable to WT animals, correlated with a lower expression of NK cell activation markers such as IFN $\gamma$, Granzyme, and CCL5 determined using qRT-PCR analysis in FACSaria isolated $\mathrm{NK} 1.1^{+}$cells (Figure 2g). Overall, TRAIL deficiency efficiently restored the number and activation status of NK cells; $\mathrm{NK} 1.1^{+} / \mathrm{CD}^{-}$cells in $\mathrm{GNMT}^{-1-}$ mice that reached comparable levels to WT animals (Figures $2 \mathrm{f}$ and $\mathrm{g}$ ). These data support the implication of TRAIL-producing NK cells in mediating liver injury and fibrogenesis in the pathological context of reduced GNMT expression.

\section{Extensive Fibrosis and Liver Damage in GNMT ${ }^{-1-}$ Mice after BDL Concurs with Activation of NK1.1 $1^{+}$Cells}

The significant attenuation of the progression of chronic liver disease and, accordingly, the lower fibrosis/cirrhosis observed in TRAIL $^{-1-} / \mathrm{GNMT}^{-1-}$ mice (Figure 2) may seem conflicting with the antifibrogenic characteristic
TRAIL-producing NK cells described by others., ${ }^{9,10}$ Thus, with the aim to elucidate the contribution of TRAILproducing NK cells to fibrogenesis, we used a well-established animal model of fibrogenesis: BDL.

First, we found that only $25 \%$ of $\mathrm{GNMT}^{-1-}$ mice survived the BDL up to 21 days, whereas survival was $60 \%$ in GNMT WT animals $\left(\mathrm{GNMT}^{+/+}\right.$; Supplementary Figure 1A). The increased activation of HSCs and abundant collagen deposition found in $\mathrm{GNMT}^{-1-}$ mice were evidenced by aSMA IF (Figure 3a) and Sirius Red staining (Figure $3 \mathrm{~b}$ ) in liver sections and further quantification was carried out at 14 and 21 days after BDL (Supplementary Figure 1B). qPCR analysis of $\alpha$ SMA and collagen1A1 expression confirmed the increased pro-fibrogenic response in $\mathrm{GNMT}^{-1-}$ mice after BDL in comparison with WT animals (Figure 3c). Interestingly, BDL led to significant increase of TLR9 in $\mathrm{GNMT}^{-1-}$ mice (Figure 3c), a receptor involved in the activation of HSC after engulfment of apoptotic hepatocyte-DNA. ${ }^{7}$

Importantly, the increased fibrogenesis observed in $\mathrm{GNMT}^{-1-}$ mice correlated with strong activation of NK1.1 ${ }^{+}$cells. Thus, FACS analysis revealed a lower presence of NK1.1 ${ }^{+}$cells in livers from $\mathrm{GNMT}^{-/-}$mice 14 days after BDL (Figure 3d), which denotes their activation. ${ }^{4}$ This was supported by the 
a

$\mathrm{GNMT}^{+/+}$

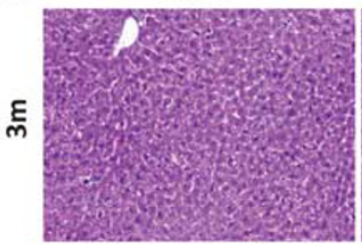

ตั

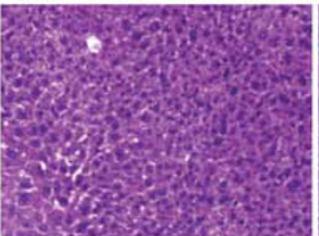

b

$\mathrm{GNMT}^{+/+}$
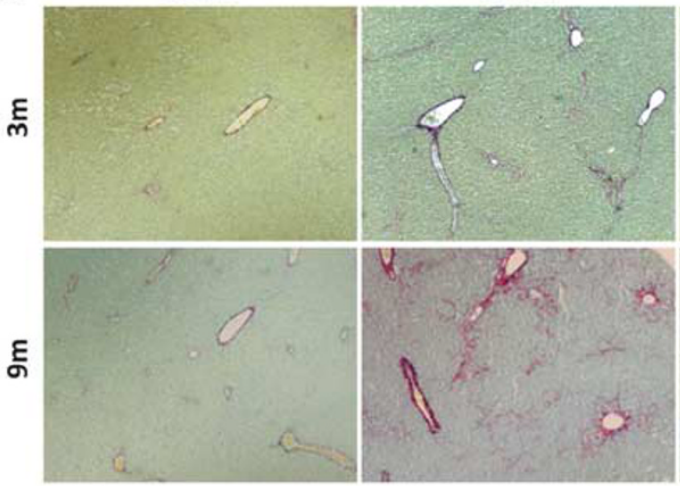

TRAIL $\% /$ GNMT $\%$
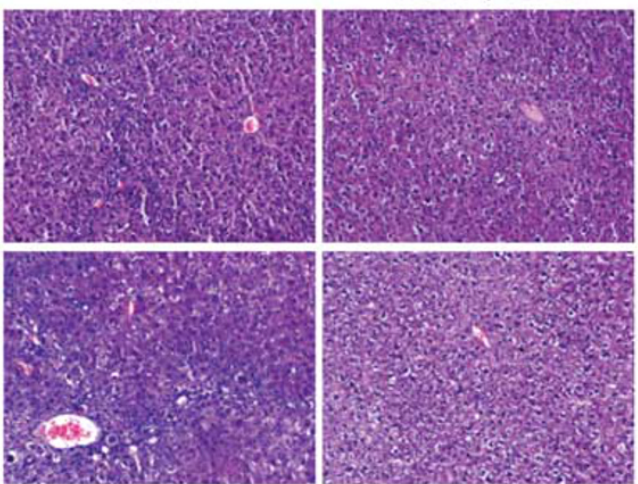

GNMT $\%$



TRAIL $\% /$ GNMT $\%$



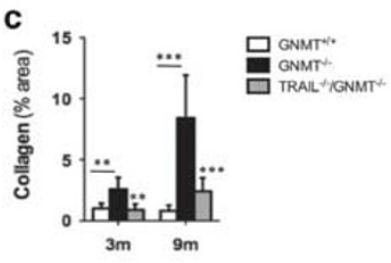
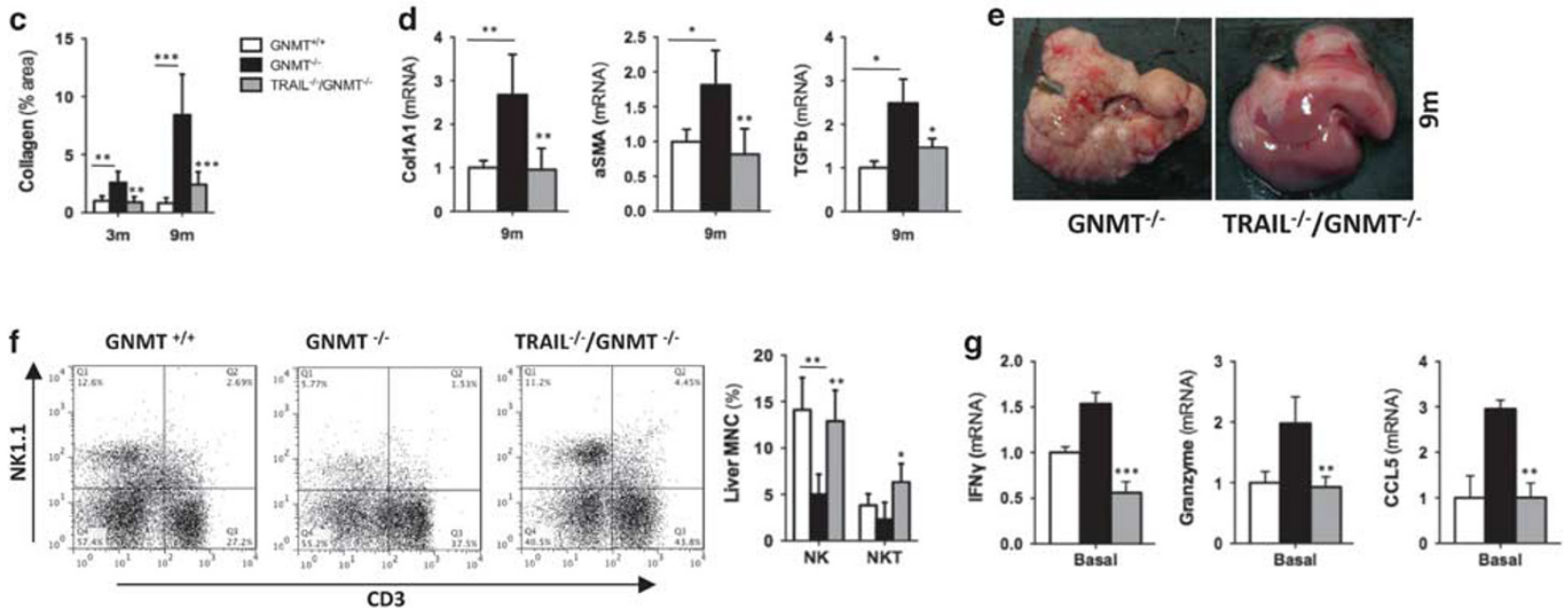

Figure 2 TRAIL deficiency reverts the spontaneous damaging phenotype in GNMT ${ }^{-1-}$ mice, which show attenuated NK cell activation. (a) H\&E staining and (b) Sirius Red staining and (c) further quantification using the FRIDA software showing attenuation of liver injury and fibrogenesis in TRAIL $^{-1-} / \mathrm{GNMT}^{-1-}$ mice compared with GNMT ${ }^{-1-}$ at 3 and 9 months of age. (d) qRT-PCR analysis showing increased Collagen1A1, $\alpha$ SMA, and TGF $\beta$ expression in 9-month-old $\mathrm{GNMT}^{-1-}$ mice compared with TRAIL $^{-1-} / \mathrm{GNMT}^{-1}$ - mice that show comparable levels to WT animals. (e) Macroscopic view of livers from $\mathrm{GNMT}^{-1-}$ and TRAIL ${ }^{-/-} / \mathrm{GNMT}^{-1-}$ mice evidencing apparent regression of the damaging phenotype in the 9 months of age double$\mathrm{KO}$ animals. (f) Dot plot of FACS analysis and bar plot showing restoration of the number of NK1.1 $1^{+}$cells in TRAIL ${ }^{-1-} / \mathrm{GNMT}^{-1-}$ mice compared with $\mathrm{GNMT}^{-1-}$ animals, all 3-month-old. Bar graph: NK cells GNMT ${ }^{+1+}$ vs $\mathrm{GNMT}^{-1-},{ }^{* *} P<0.01$; NK cells $\mathrm{GNMT}^{-1-} \mathrm{vs} \mathrm{GNMT}^{-1-} / \mathrm{TRAIL}^{-1-}$, **P $<0.01$; NKT cells, ${ }^{*} P<0.05$. (g) qPCR analysis on FACSaria isolated NK1.1 ${ }^{+}$cells confirming low activation in TRAIL ${ }^{-1-} / \mathrm{GNMT}^{-1-}$ cells compared with $\mathrm{GNMT}^{-\prime-}$ cells. $n=5-10 .{ }^{*} P<0.05 ;{ }^{* *} P<0.01,{ }^{* *} P<0.001$ (GNMT $^{-\prime-}$ vs $\mathrm{TRAIL}^{-1-} / \mathrm{GNMT}^{-1}{ }^{-}$). Error bars represent s.d.

higher expression of TRAIL found in liver NK1.1 ${ }^{+}$cells from $\mathrm{GNMT}^{-1-}$ mice after BDL (Figure 3e). In addition, the elevated expression of granzyme and perforin, the mediators of
NK1.1 ${ }^{+}$cell-mediated cell death, and RANTES/CCL5 found in $\mathrm{GNMT}^{-1-}$ mice after BDL confirmed the activation of these cells (Supplementary Figure 1C). We previously 
a
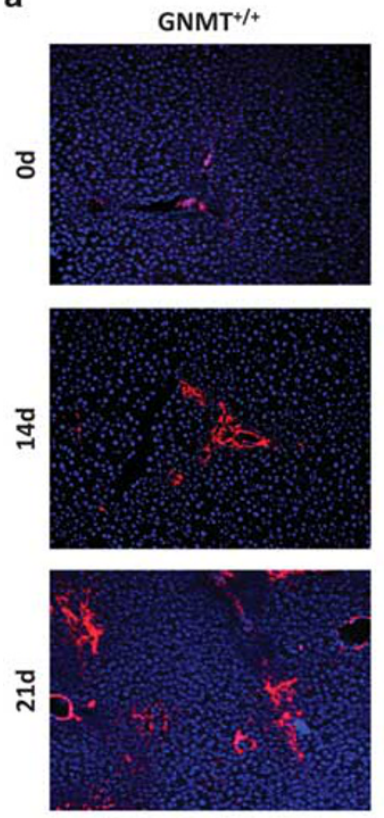
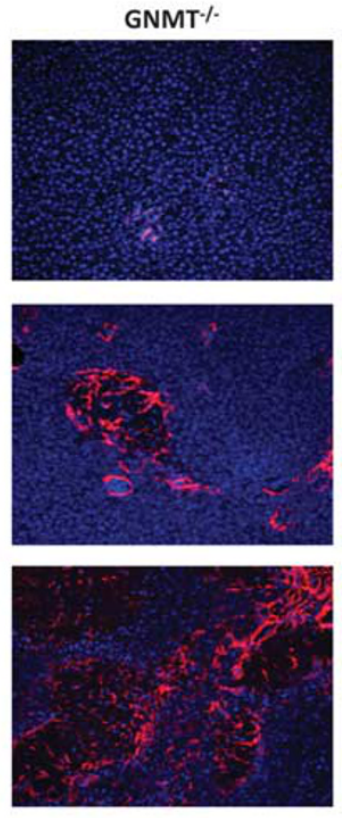

b
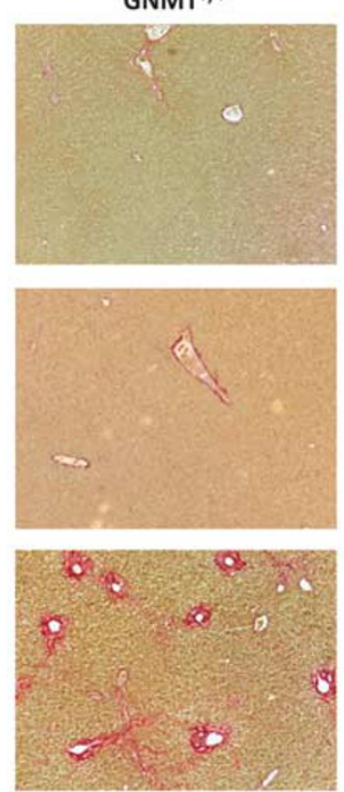

GNMT $\%$
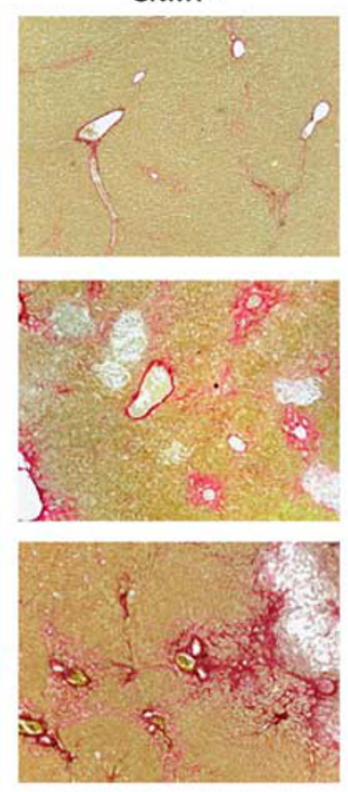

C
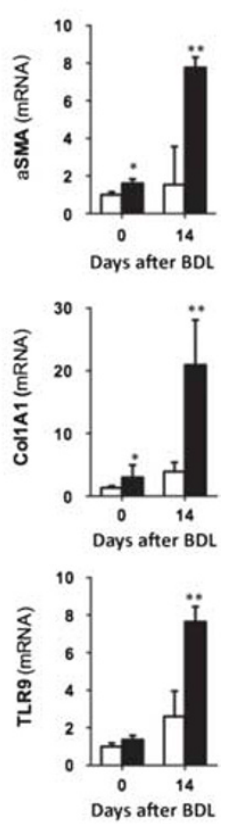

d

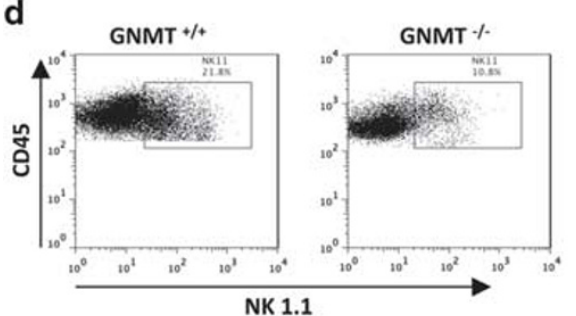

e
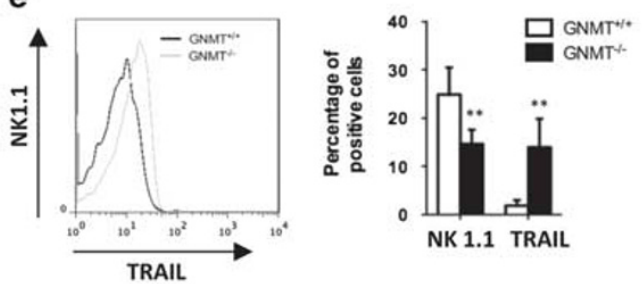

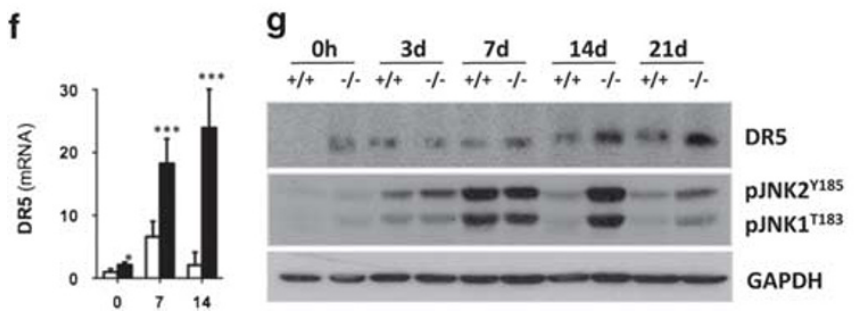

Figure 3 Exacerbated fibrogenesis in GNMT ${ }^{-1-}$ mice concurs with activation of NK1.1 ${ }^{+}$cells (a) IF using an $\alpha$ SMA Ab and (b) Sirius Red staining showed increased fibrosis in GNMT ${ }^{-1-}$ mice. (c) qPCR analysis of mRNA expression of $\alpha$ SMA, collagen1A1, and TLR9 confirmed HSC activation. (d) Dot plot of FACS analysis on isolated liver mononuclear cells (MNCs) showing lower number of $\mathrm{CD} 45^{+} / \mathrm{NK} 1.1 .^{+}$cells in GNMT ${ }^{-1-}$ mice. (e) Histogram showing higher presence of TRAIL in the cell surface of NK1.1 $1^{+}$cells in GNMT ${ }^{-1-}$ liver MNCs. Bar plot representing the data obtained by FACS analysis showing low presence of NK1.1 $1^{+}$cells correlating with high expression of TRAIL in GNMT ${ }^{-1-}$ liver MNCs. (f) qPCR analysis showing increased DR5 mRNA expression in $\mathrm{GNMT}^{-1-}$ mice. (g) Western blot analysis confirmed that GNMT ${ }^{-1-}$ mice have higher expression of DR5 after BDL correlating with increased phosphorylation of JNK after surgery. $n=5-7 .{ }^{*} P<0.05 ;{ }^{* *} P<0.01 ; * * * P<0.001\left(\mathrm{GNMT}^{+/+}\right.$vs GNMT $^{-/-}$). Error bars represent s.d.

described that NK cells were cytotoxic against $\mathrm{GNMT}^{-/-}$ hepatocytes and actively contribute to liver injury. ${ }^{4}$ Accordingly, increased liver injury in $\mathrm{GNMT}^{-1-}$ mice after BDL was evidenced by high-serum transaminases and bilirubin levels (Supplementary Figure 1D) and H\&E staining on liver sections showing wide necrotic areas (Supplementary Figure 1E, G). The increased apoptosis in $\mathrm{GNMT}^{-1-}$ mice after BDL compared with WT mice was evidenced using TUNEL assay (Supplementary Figure 1F) and quantification of caspase-3 activity (Supplementary Figure 1G, lower panel). Importantly, increased NK1.1 ${ }^{+}$cell activation and liver injury in $\mathrm{GNMT}^{-1-}$ mice correlated with higher mRNA and protein expression of DR5 observed in $\mathrm{GNMT}^{-1-}$ mice, which may contribute to the cytotoxicity of TRAILproducing $\mathrm{NK} .1^{+}$cells after BDL (Figures $3 \mathrm{f}$ and $\mathrm{g}$ ). In accordance, strong and sustained phosphorylation of JNK, the downstream kinase activated upon DR5 engagement, was found in $\mathrm{GNMT}^{-1-}$ animals after BDL (Figure 3g). 
Tissue destruction triggers the inflammatory response in an attempt to remove cell debris, mainly mediated by KCs. Previous work highlighted the implication of $\mathrm{KC}$ during fibrogenesis in different experimental models. ${ }^{20,21}$ Interestingly, despite the more severe liver injury and fibrosis observed in $\mathrm{GNMT}^{-1-}$ mice we found lower presence of F4/80-positive cells after BDL compared with WT animals (Supplementary Figure $1 \mathrm{H}$ ). Finally, TNF serum levels were lower in $\mathrm{GNMT}^{-1-}$ mice compared with WT after 14 days supporting the low activation of KC observed (Supplementary Figure 1I). These data are consistent with the lower KC activation described in $\mathrm{GNMT}^{-/-}$mice in response to LPS, where NK cells but not KC mediated endotoxin-liver damage. ${ }^{4}$

\section{Inhibition of NK1.1 ${ }^{+}$Cells Protects the Liver against Injury and Fibrogenesis after BDL in the Absence of GNMT}

$\mathrm{GNMT}^{-1-}$ mice show increased NK1.1 ${ }^{+}$cell activation that coincides with increased fibrogenesis after BDL (Figure 3). NK1.1 is a cell surface marker of both NK and NKT cells, which have shown opposing roles during fibrogenesis. ${ }^{8,10}$ Our data point to a role of TRAIL-producing NK cells in mediating liver injury and fibrogenesis in the absence of GNMT (Figure 2). Therefore, with the aim to better delineate the implication of NK cells during this process we performed two experimental approaches: (i) we inhibited the whole $\mathrm{NK} 1.1^{+}$cell compartment using an NK1.1-depleting antibody and (ii) we specifically depleted NK cells using the antiASIALO-GM1 antibody (ASIALO) before and after BDL.

The important beneficial impact of NK1.1 ${ }^{+}$cell depletion was evidenced by the $100 \%$ survival of NK1.1/GNMT ${ }^{-1-}$ mice after $\mathrm{BDL}$, in clear contrast to the high mortality observed in $\mathrm{GNMT}^{-1-}$ mice (Figure 4a). Lower serum transaminase levels improved liver histology showing less necrotic areas, reduced presence of TUNEL-positive cells and lower caspase-3 activity confirmed the beneficial impact of $\mathrm{NK} 1.1^{+}$cell depletion on attenuating liver injury in $\mathrm{GNMT}^{-1-}$ mice after BDL (Figures $4 \mathrm{~b}-\mathrm{d}$ ). In accordance with reduced cell death, liver fibrosis was significantly attenuated in NK1.1/GNMT ${ }^{-1-}$ mice as evidenced by $\alpha$ SMA IF, Sirius Red staining, further quantification and qPCR of aSMA and collagen $1 \mathrm{~A} 1$ expression (Figures $4 \mathrm{e}-\mathrm{g}$ ). In addition, the decreased expression of TLR9 found in NK1.1/ $\mathrm{GNMT}^{-/-}$mice after BDL further supported the lower activation of HSC (Figure 4g).

$\mathrm{KC}$ activation was partially restored in NK1.1/GNMT ${ }^{-1-}$ mice as shown by F4/80 IHC and TNF serum levels (Supplementary Figure 2A, B), suggesting an inhibitory crosstalk between KC and NK cells in the absence of GNMT. Finally, inhibition of $\mathrm{NK}^{+} .1^{+}$cells in $\mathrm{GNMT}^{-1-}$ mice correlated with lower DR5 expression and less activation of JNK after BDL (Figure $4 \mathrm{~g}$ and $\mathrm{h}$ ).

Overall, our data suggest that NK1.1 ${ }^{+}$cell activation leads to increased liver injury, strongly contributing to fibrogenesis after BDL in the context of GNMT absence.
Selective Inhibition of TRAIL-Producing NK Cells has an Antifibrogenic Effect after BDL in the Absence of GNMT NK cells are described as the main producers of TRAIL in the liver. ${ }^{22,23}$ Here we provide further evidence that NK cells isolated from $\mathrm{GNMT}^{-1-}$ mice express significantly more TRAIL than NKT cells (Supplementary Figure 3A). Moreover, we also show that NK cells from $\mathrm{GNMT}^{-1-}$ mice express more TRAIL than $\mathrm{GNMT}^{+/+}$animals both using FACS analysis and qRT-PCR analysis in FASCaria isolated cells (Supplementary Figure 3B, C), further supporting the potential implication of TRAIL-producing NK cells in the pathogenesis of $\mathrm{GNMT}^{-1-}$ mice.

Next, in order to better delineate the implication of TRAIL-producing NK cells during fibrogenesis, we specifically depleted this cell compartment using the anti-ASIALOGM1 antibody (ASIALO). Similarly to what we found in NK1.1/GNMT ${ }^{-1-}$ animals, liver fibrosis was significantly attenuated in ASIALO/GNMT ${ }^{-1-}$ mice as evidenced by $\alpha \mathrm{SMA}$, Sirius Red staining, and further quantification (Figures $5 \mathrm{a}$ and $\mathrm{b}$ ). $\mathrm{qPCR}$ of $\alpha \mathrm{SMA}$ and collagen 1A1 expression confirmed the reduced fibrosis found in ASIALO/ $\mathrm{GNMT}^{-1-}$ mice after BDL. In addition, the decreased expression of TLR9 found in ASIALO/GNMT ${ }^{-1-}$ mice after BDL pointed to a lower activation of HSC (Figure 5b). The beneficial impact of specific inhibition of NK cells was supported by the high survival rate in ASIALO/GNMT ${ }^{-1-}$ mice compared with $\mathrm{GNMT}^{-1-}$ animals (Figure 5c). Further analysis of serum transaminases (Figure 5d), H\&E staining, and TUNEL assay (Figures $5 e$ and $\mathrm{f}$ ) confirmed the significant improvement of the liver parenchyma status and the attenuation of cell death in NK cell-depleted mice after BDL. ASIALO/GNMT ${ }^{-1-}$ mice showed reduced DR5 expression and attenuated activation of JNK 14 days after BDL (Figures $5 \mathrm{~g}$ and h). Finally, ASIALO/GNMT ${ }^{-1-}$ mice showed partial recovery of $\mathrm{KC}$ activation evidenced by F4/80 IHC and TNF ELISA (Supplementary Figure 4A, B), supporting the regulation between cell compartments in the context of cholestatic-liver damage in $\mathrm{GNMT}^{-1-}$ animals.

In parallel, a control group receiving IgG showed no significant differences in fibrogenesis and liver injury when compared with the uninjected $\mathrm{GNMT}^{-1-}$ mice, overall proving that the latter group can be considered as a proper control for both $\mathrm{NK} 1.1^{+}$and ASIALO-treated groups (Supplementary Figure 5).

Overall, our data point to the implication of TRAIL-producing NK cells in mediating BDL-induced liver injury and further fibrogenesis in the absence of GNMT.

\section{TRAIL Deficiency Attenuates Fibrogenesis in GNMT ${ }^{-1-}$ Mice but Exacerbates the Necrotic Response after BDL}

NK cells are the main source of TRAIL (Supplementary Figure 3), which mediates their antifibrogenic effect by promoting HSC death. ${ }^{10}$ Conversely, previous work described that TRAIL has a main role in mediating cholestatic liver 


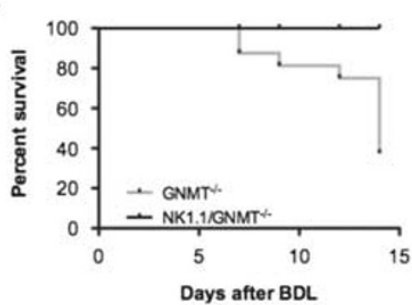

C
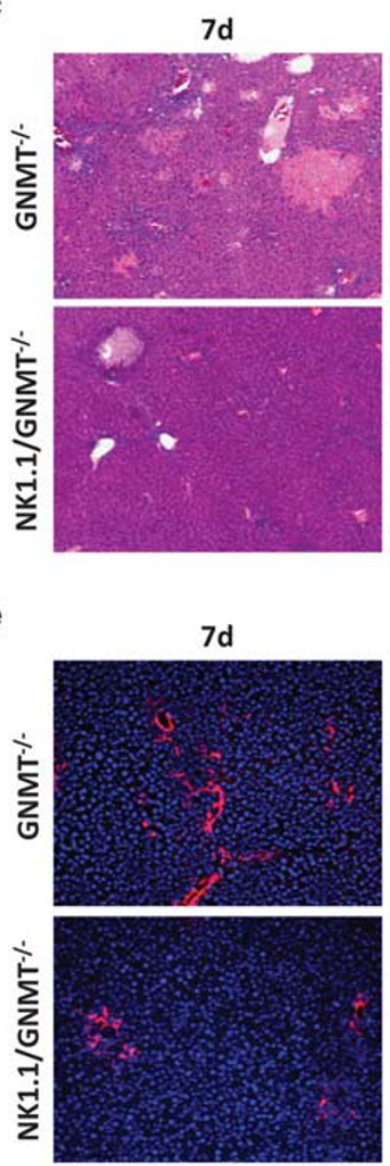

g

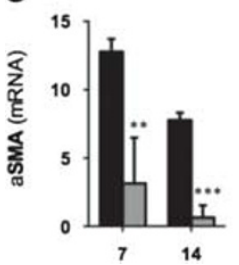

b
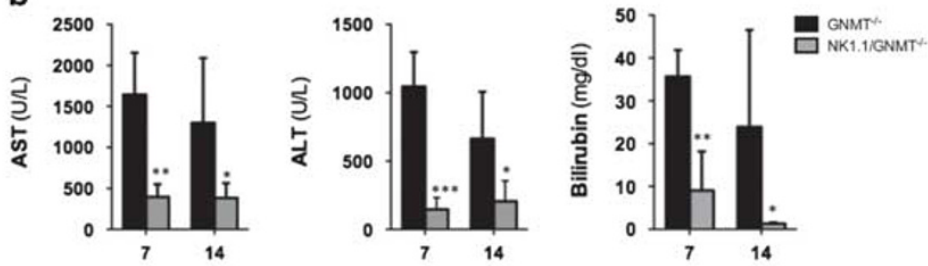

d
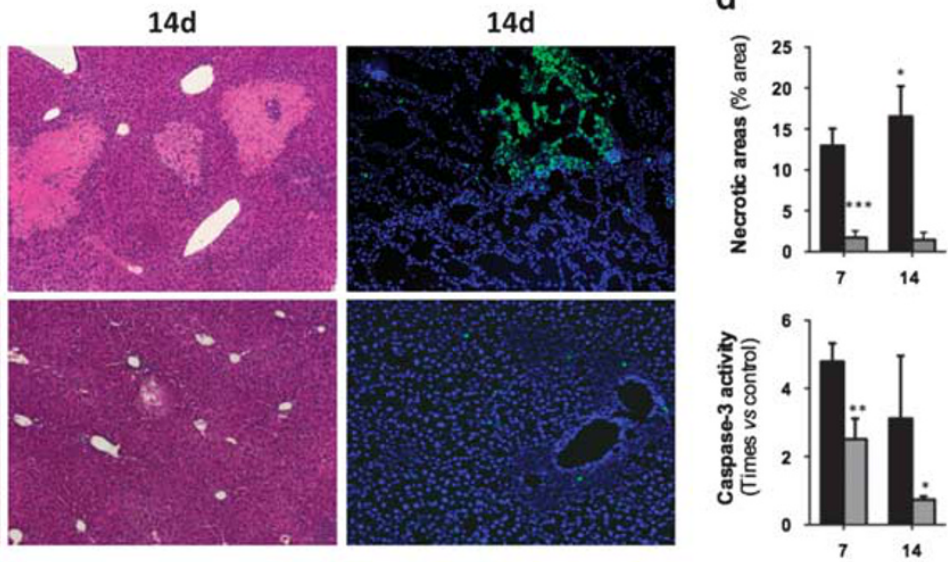

$7 d$
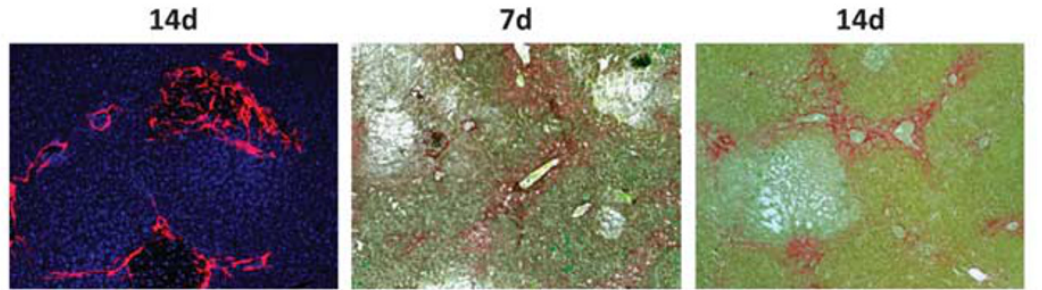

f
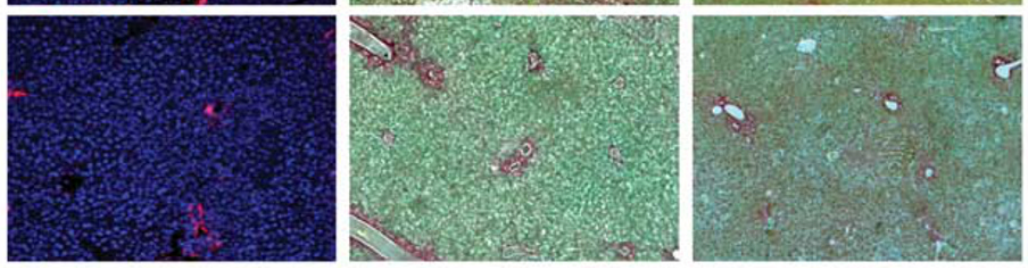

h
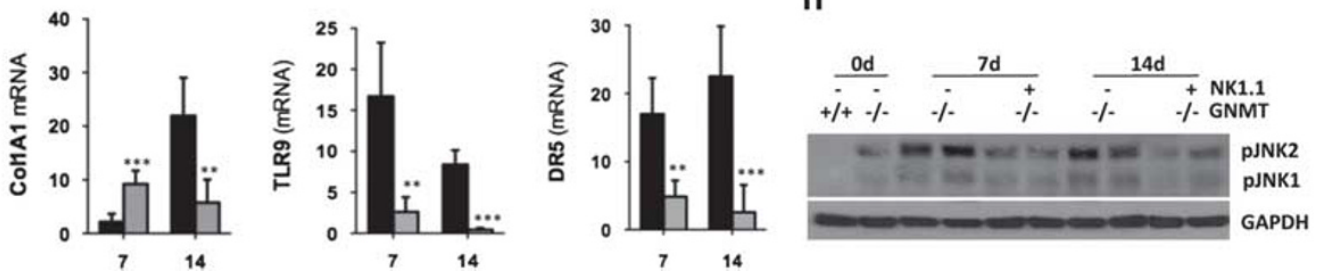

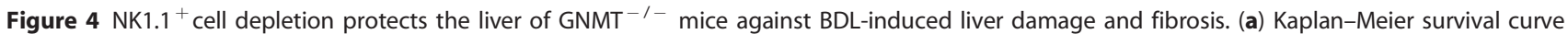
evidencing the beneficial impact of NK1.1 ${ }^{+}$cell depletion after BDL. (b) Serum transaminase and billirubin analysis, (c) H\&E staining, and TUNEL assay on liver sections showing lower liver injury after BDL in NK1.1/GNMT ${ }^{-/}$mice. (d) Further quantification of necrotic areas on H\&E sections and caspase-3 activity on whole-liver extracts confirmed these data. (e) IHC using an $\alpha$ SMA Ab and Sirius Red staining and (f) further quantification evidenced reduced fibrosis in NK1.1/GNMT ${ }^{-1-}$ mice. (g) qPCR analysis of $\alpha$ SMA, collagen1A1, TLR9, and DR5 expression. (h) WB analysis showing lower pJNK expression in NK1.1/GNMT ${ }^{-1-}$ mice after BDL. $n=5-7 .{ }^{*} P<0.05 ; * * P<0.01 ; * * * P<0.001$ (GNMT $^{-1-}$ vs NK1.1/GNMT ${ }^{-1-}$ ). Error bars represent s.d.

injury. ${ }^{14}$ In this line, here we describe that TRAIL depletion notably attenuated the chronic damaging phenotype found in $\mathrm{GNMT}^{-1-}$ mice (Figure 2). Finally, inhibition of NK1.1 $1^{+}$ cells and specifically of TRAIL-producing NK cells efficiently protected the liver against severe injury and fibrosis in GNMT $^{-/-}$mice after BDL (Figures 4 and 5). 

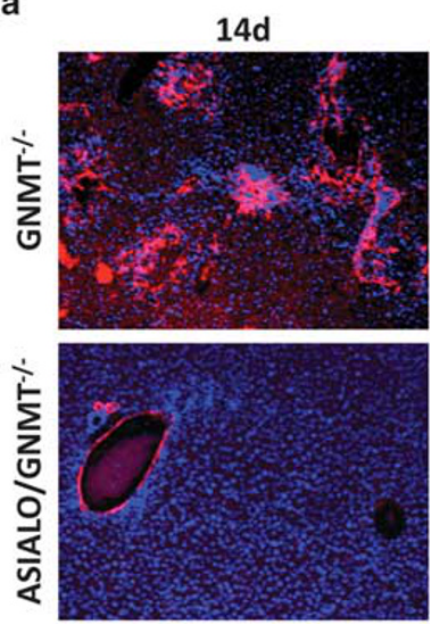

C
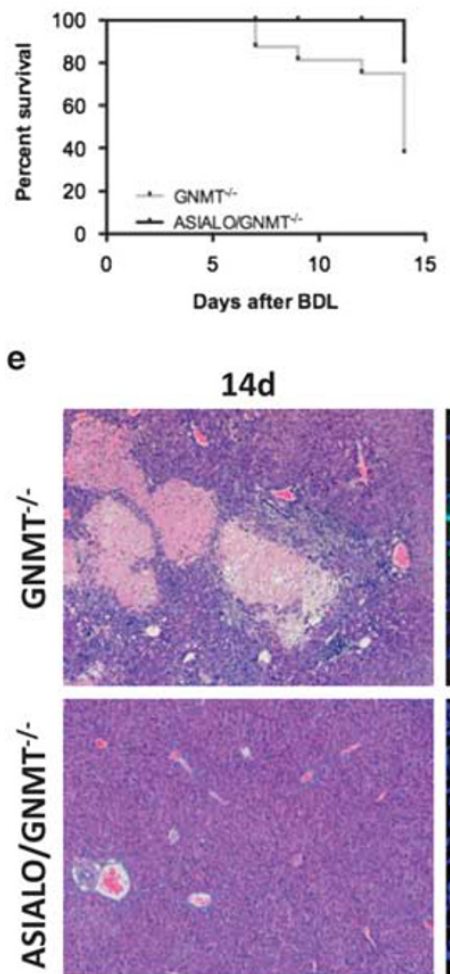

14d
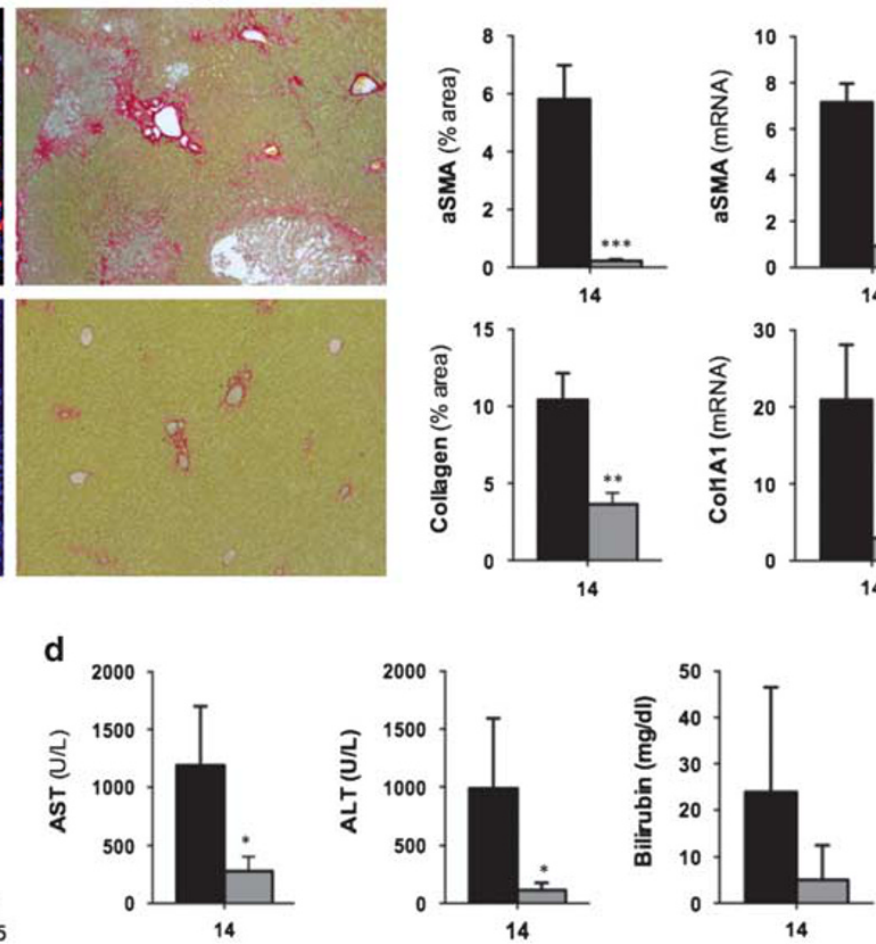

$\mathbf{f}$ $14 d$
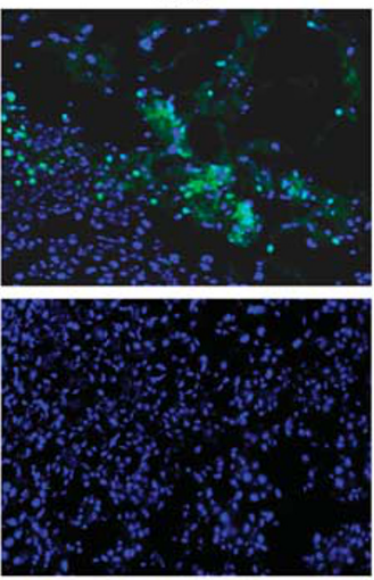
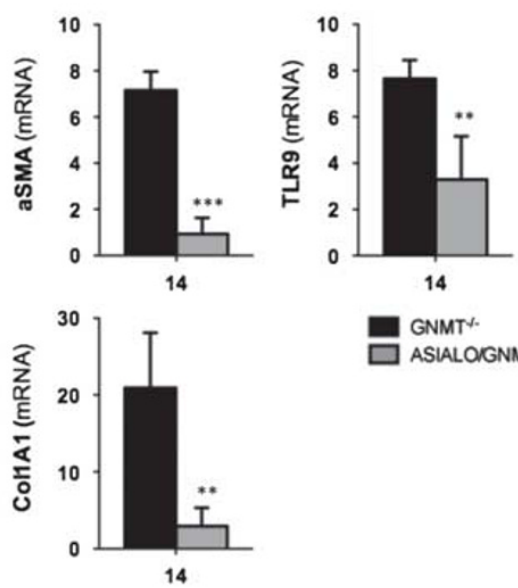

b

GNMT*

ASIALOGNMT

Figure 5 Specific NK cell inhibition with ASIALO protects the liver of GNMT ${ }^{-1-}$ mice from BDL-liver injury and fibrosis. (a) $\alpha$ SMA IF (left panels) and Sirius red staining (right panels), further quantification using the FRIDA software and (b) qPCR of $\alpha$ SMA, collagen1A1, and TLR9 expression evidenced the significantly lower fibrosis in ASIALO/GNMT ${ }^{-1-}$ after BDL. (c) Kaplan-Meier survival curve showing improved animal survival in ASIALO/GNMT ${ }^{-/-}$ mice compared with GNMT ${ }^{-1-}$ animals after BDL. (d) Analysis of serum transaminases and bilirubin, (e) H\&E staining (left panels), and TUNEL assay (right panels) showed an evident reduction of liver injury in ASIALO/GNMT ${ }^{-/-}$mice after BDL. (f) Quantification of necrotic areas in H\&E-stained liver sections after BDL showing less necrosis in ASIALO/GNMT ${ }^{-1-}$ mice. (g) qPCR analysis of DR5 expression on liver samples and (h) lower phosphorylation of JNK detected with western blot analysis evidenced lower activation of the DR5-mediated cell death response in ASIALO/GNMT ${ }^{-/-}$ mice after BDL. $n=5-7 .{ }^{* *} P<0.01\left(\mathrm{GNMT}^{-/-}\right.$vs ASIALO/GNMT ${ }^{-{ }^{-}}$). Error bars represent s.d.

Overall, in order to better delineate the implication of TRAIL during fibrogenesis in the pathological context of GNMT deficiency we next performed BDL in $\mathrm{TRAIL}^{-/-}$, $\mathrm{GNMT}^{-/-}$mice. Liver fibrosis was significantly attenuated in TRAIL $^{-1-} / \mathrm{GNMT}^{-1-}$ animals when compared with GNMT $^{-1-}$ mice 21 days after BDL (Figures 6a-c, Supplementary Figure 6A). In addition, TLR9 was significantly less expressed in the double-KO mice, supporting lower activa- 
tion of HSC after BDL (Supplementary Figure 6B). Surprisingly, despite the obvious attenuation in liver fibrosis exerted by TRAIL depletion, BDL led to a similar lethality of TRAIL $^{-1-} /$ GNMT $^{-1-}$ mice, mainly occurring within the first days after surgery (Figure 6d). Further analysis showed that mortality of $\mathrm{TRAIL}^{-1-} / \mathrm{GNMT}^{-1-}$ mice correlated with severe liver necrosis, especially at 3 days after BDL (Figures $6 \mathrm{e}$ and $\mathrm{g}$ upper panel). Interestingly, hepatocyte apoptosis was attenuated at 3,7 , and 14 days after BDL in TRAIL $^{-1-} /$ GNMT $^{-1-}$ mice (Figure $6 f$ and g lower panel). Accordingly, with increased necrosis and lower apoptosis, we found less FADD expression in $\mathrm{TRAIL}^{-1-} / \mathrm{GNMT}^{-1-}$ mice after BDL when compared with $\mathrm{GNMT}^{-1-}$ and WT animals
(Figure 6h). In addition, we observed reduced expression of DR5 in livers from the double-KO mice after BDL (Figure 6i).

In vitro assays confirmed the higher susceptibility to necrosis conferred by TRAIL depletion in $\mathrm{GNMT}^{-1-}$ mice. Thus, isolated hepatocytes from TRAIL $^{-1-} / \mathrm{GNMT}^{-1-}$ mice showed lower survival after deoxycholic acid treatment, similar to $\mathrm{GNMT}^{-1-}$ cells, when compared with cultured $\mathrm{GNMT}^{+/+}$cells (Supplementary Figure 7A). Interestingly, despite comparable low survival, caspase-3 activity in TRAIL $^{-1-} / \mathrm{GNMT}^{-1-}$ cells was significantly lower than in GNMT $^{-1-}$ hepatocytes after DCA and comparable to WT cells (Supplementary Figure 7B). Finally, we found higher
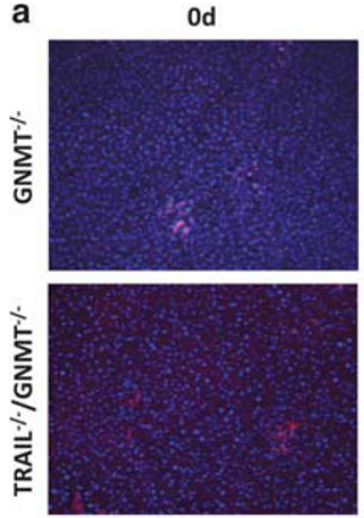

d

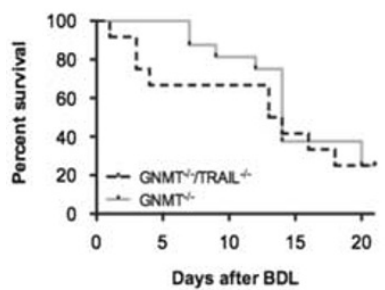

g
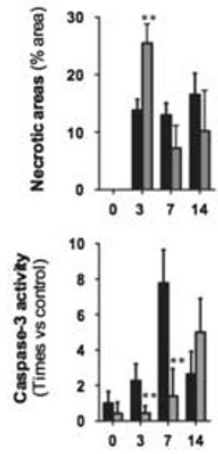

21d
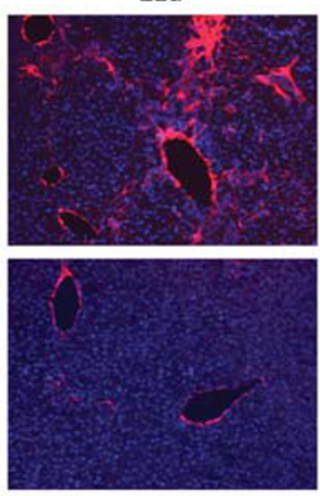

e


Od
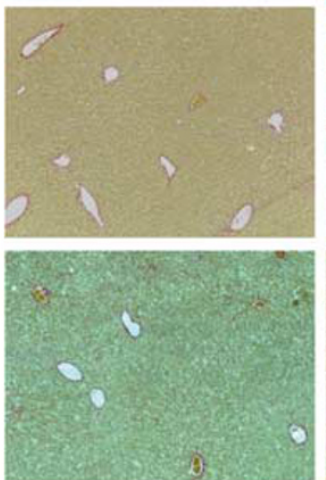

7d 21d

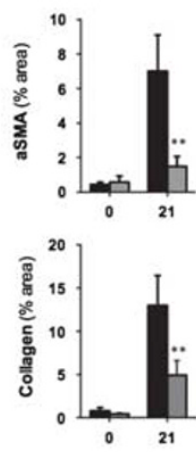

f

$7 d$
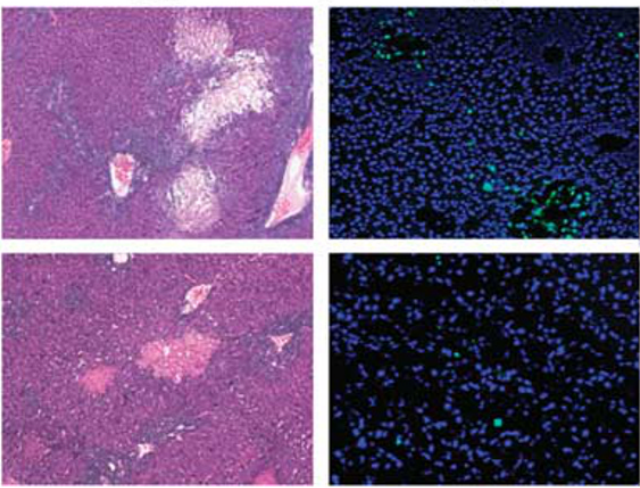

i

h $\frac{O d}{W_{T} G^{\%} / T / G} \frac{3 d}{W T} \quad G^{\%} \quad$ T/G $\%$

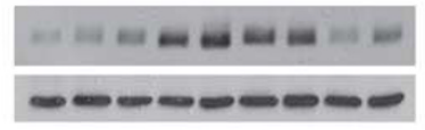

FADD

GAPDH

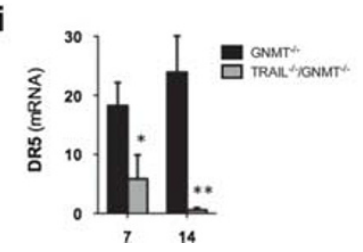

Figure 6 TRAIL deficiency in GNMT ${ }^{-/-}$mice attenuates fibrogenesis after BDL but shifts cell death from apoptosis to necrosis. (a) TRAIL ${ }^{-/-} /$ $\mathrm{GNMT}^{-/-}$mice show significantly lower fibrosis than GNMT ${ }^{-/-}$mice after BDL as shown by IF using an $\alpha$ SMA Ab, Sirius Red staining, and further quantification. (b) Kaplan-Meier curve showing an overall comparable death rate after BDL in $\mathrm{TRAIL}^{-/-} / \mathrm{GNMT}^{-/-}$mice compared with GNMT $^{-/-}$animals. (c) H\&E staining and (g) further quantification (upper panel), (d) TUNEL assay and (g) Caspase3 activity (lower panel) of liver extracts showing lower apoptotic cell death but profuse necrosis in TRAIL ${ }^{-/} / \mathrm{GNMT}^{-/}$- mice after BDL. (h) WB analysis showing lower FADD in TRAIL ${ }^{-/-} /$ $\mathrm{GNMT}^{-/-}$mice after BDL. (i) qPCR showing lower DR5 expression in $\mathrm{TRAIL}^{-/-} / \mathrm{GNMT}^{-/}-$mice $^{14}$ days after BDL. $n=5-7$. ${ }^{*} P<0.05 ;{ }^{*} P<0.01$; ***P $<0.001$ (TRAIL $^{-/-} / \mathrm{GNMT}^{-/-}$vs $\mathrm{GNMT}^{-/-}$). 
expression of RIP1 and RIP3 in $\mathrm{TRAIL}^{-/-} / \mathrm{GNMT}^{-/-}$cells exposed to DCA compared with $\mathrm{GNMT}^{-1-}$ cells (Supplementary Figure 7C).

Overall, our data show that TRAIL deficiency in $\mathrm{GNMT}^{-1-}$ hepatocytes promotes a shift from apoptosis toward necrotic cell death. Moreover, our data suggest that TRAIL has a main role during fibrogenesis by mediating hepatocyte apoptosis in the pathogenic context of GNMT deficiency.

\section{In vivo Silencing of DR5 Protects $\mathrm{GNMT}^{-1-}$ Mice against BDL-Induced Liver Injury}

Finally, silencing of DR5 in GNMT ${ }^{-1-}$ mice confirmed the implication of the TRAIL/DR5 axis in mediating liver injury and further fibrogenesis. Thus, fibrosis was significantly reduced in ShDR5/GNMT ${ }^{-1-}$ mice as we found lower $\alpha$ SMA levels and collagen deposition after BDL when compared with $\mathrm{GNMT}^{-1-}$ mice (Figure $7 \mathrm{a}$ ). Low fibrogenesis after DR5 silencing correlated with an overall significant improvement in the liver parenchyma of ShDR5/GNMT ${ }^{-1-}$ mice that showed significantly lower AST, ALT, and bilirubin serum levels after BDL (Figure 7b). H\&E staining, TUNEL assay, caspase- 3 activity, and a survival rate of $90 \%$ confirmed the liver protection exerted by silencing of DR5 in $\mathrm{GNMT}^{-1-}$ mice (Figures $7 \mathrm{c}-\mathrm{e}$ ).

Overall, these data point to the hepatoprotective effect of DR5 deletion during BDL-induced liver injury in the pathogenic context of GNMT deficiency and suggest that attenuation of both DR5-mediated necrosis and apoptosis in $\mathrm{GNMT}^{-1-}$ mice protects the liver against fibrogenesis after BDL.

\section{DISCUSSION}

Chronic liver injury triggers fibrogenesis, a tissue remodeling and scarring process that leads to cirrhosis and HCC regardless of disease etiology. ${ }^{5}$ GNMT, the enzyme that metabolizes S-adenosylmethionine, is downregulated in cirrhotic patients from diverse etiologies such as HCV and alcohol, and is absent in HCC. ${ }^{2}$ Here, we show that GNMT is also downregulated during human cholestatic liver disease. Importantly, we observed that GNMT is reduced both at initial stages in patients with mild cholestasis and low degree of ductular alterations as well as in patients with more severe liver injury before transplantation. These data, together with previous work describing the lower expression of GNMT in NAFLD patients, ${ }^{24}$ support the important role of GNMT during the pathogenesis of liver disease regardless of the etiology and suggest a causative role for the deficiency of GNMT rather than a consequence of altered liver homeostasis.

In accordance, GNMT-deficient mice develop fibrosis, cirrhosis, and HCC, ${ }^{3}$ which make them a valuable tool to investigate the molecular mechanisms underlying the pathogenesis of chronic liver disease. We previously described that in $\mathrm{GNMT}^{-1-}$ mice, NK cells had a strong cytotoxic activity against autologous hepatocytes, which exhibited high expression of NK cell-activating ligands and TRAILR2/DR5, evidencing that GNMT deficiency promotes a 'transformedlike' phenotype in hepatocytes. ${ }^{4}$ In $\mathrm{GNMT}^{-1-}$ mice, NK cell activation led to increased apoptotic cell death, mainly mediated by TRAIL, which overall contributed to the inflammatory environment, distinctive of the progression of NASH. ${ }^{4}$ Nonetheless, the role of TRAIL during chronic liver disease and fibrogenesis in the pathogenic context of GNMT deficiency was not further investigated. Here, we provide evidence that NK cells from $\mathrm{GNMT}^{-1-}$ mice produce more TRAIL than WT cells and that depletion of TRAIL in GNMT $^{-1-}$ mice efficiently protects the liver against the progression of chronic liver disease characterized by profuse fibrogenesis. Importantly, the regression of the damaging phenotype in TRAIL $^{-1-} / \mathrm{GNMT}^{-1-}$ mice correlated with a lower activation of the NK1.1 ${ }^{+}$cell compartment and an overall restoration of the percentage of NK cells that reached comparable levels to WT animals.

NK and NKT cells have a differential role during fibrogenesis. Generally, whereas NKT cells are considered as pro-fibrogenic, ${ }^{8}$ NK cells are commonly described as antifibrogenic as they induce HSC death through TRAIL and RAE1/NKG2D (reviewed in ${ }^{\text {Gao25}) . ~ H o w e v e r, ~ N K ~ c e l l-~}$ mediated HSC death strongly depends on the activation status of the latter. Thus, NK cells kill early- but not fully activated HSC due to the loss of expression of RAE1 over time. ${ }^{9}$ Similarly, HSC activated by phagocytosis of apoptotic bodies are resistant to TRAIL-mediated cell death. ${ }^{26}$ Moreover, TRAIL has been described as a mediator of cholestatic liver injury in mice and a contributor to chronic cholestatic liver disease in humans through bile duct destruction. ${ }^{11-14}$ Taking all these together, the concurrence of a damaging phenotype progressing to fibrosis with strong activation/cytotoxicity of NK cells found in the context of GNMT deficiency is somewhat intriguing. Thus, we aimed to elucidate the implication of NK cells during fibrogenesis in the pathogenic context of GNMT deficiency. We convincingly show that fibrosis progresses in the context of NK cell activation and that specific inhibition of NK cells efficiently protects the liver against BDL injury/fibrosis in the absence of GNMT. Our data in TRAIL $^{-1-} / \mathrm{GNMT}^{-1-}$ mice further support the role of TRAIL during fibrogenesis in GNMT-deficient hepatocytes as mediators of hepatocyte apoptotic death. Interestingly, whereas transient amelioration of TRAIL in ASIALO/GNMT ${ }^{-/-}$mice efficiently protected the liver against BDL-acute injury, persistent blockade of apoptosis in TRAIL $^{-1-} / \mathrm{GNMT}^{-1-}$ mice promoted a high susceptibility to BDL-induced necrotic cell death. The shift from apoptosis to necrotic cell death is in accordance with previous work showing that persistent inhibition of apoptosis through genetic caspase-8 depletion sensitizes the liver to RIP1/3-mediated necrotic cell death. ${ }^{27}$ The expression of FADD; the endogenous inhibitor of RIP-mediated necrosis ${ }^{28}$ was found to be low in $\mathrm{TRAIL}^{-1-} / \mathrm{GNMT}^{-1-}$ livers, 
a
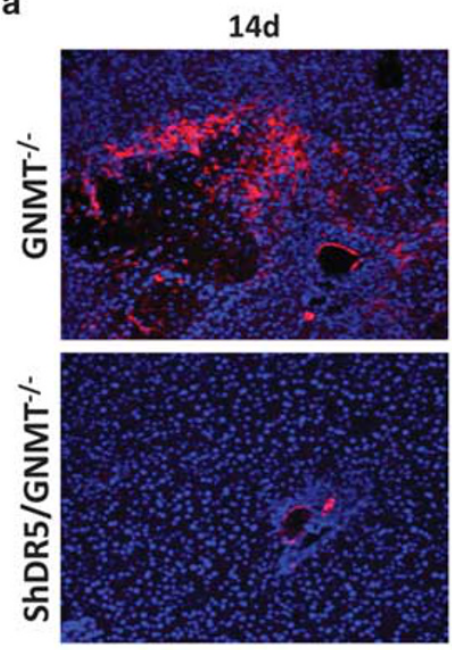

$14 d$
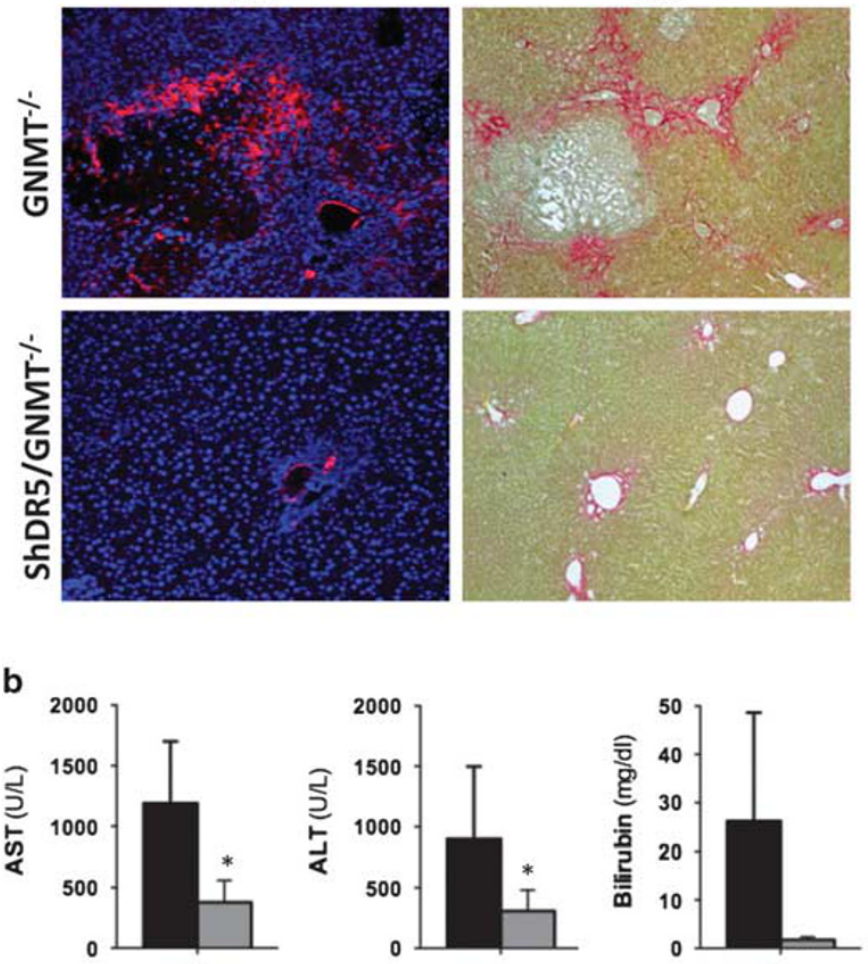

14

C

$14 d$
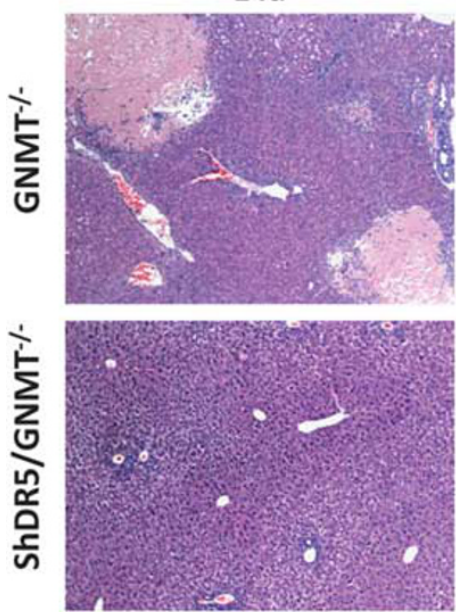

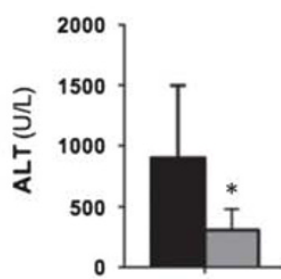

14

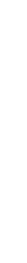

d
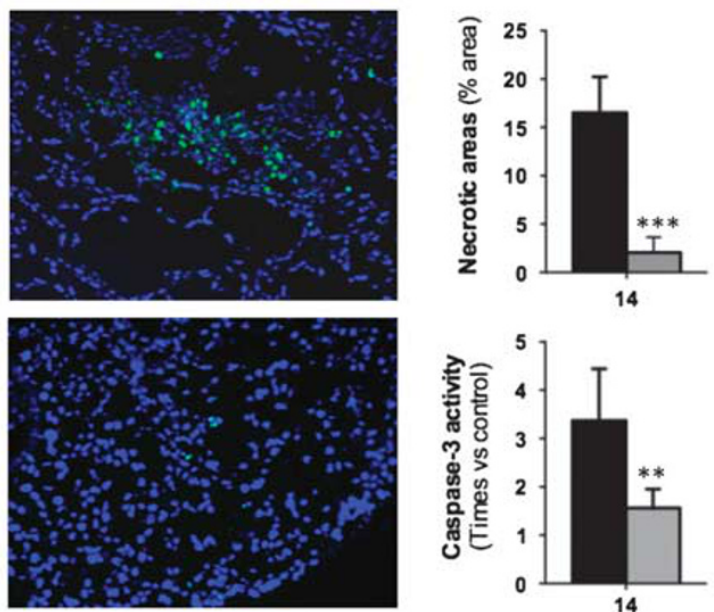

14d
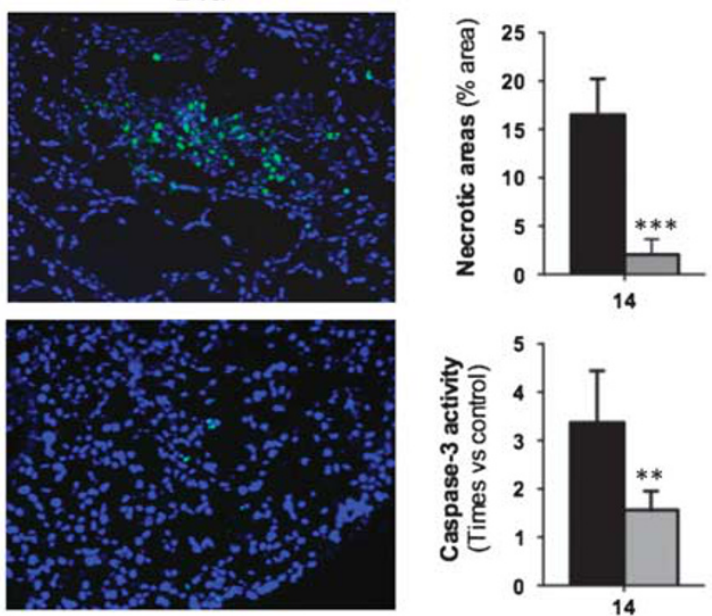


$\mathrm{GNMT}^{*}$

ShDR5/GNMT ${ }^{*}$

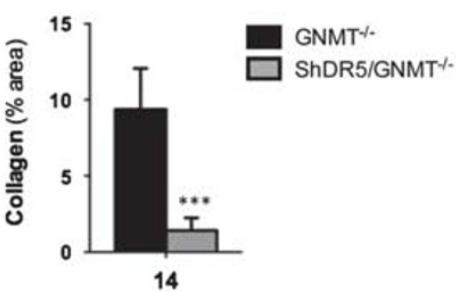

e

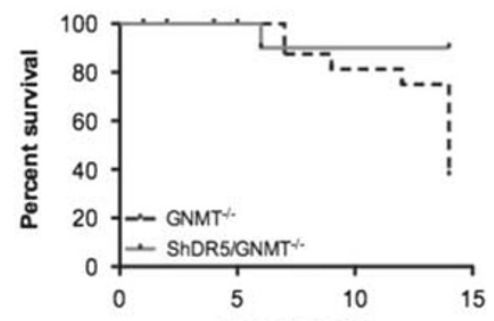

Days after BDL

Figure 7 In vivo silencing of DR5 in GNMT ${ }^{-1-}$ mice attenuates fibrosis protecting the liver against BDL-induced injury. (a) IF using $\alpha \mathrm{SMA}$ Ab and Sirius Red staining and qPCR analysis of $\alpha$ SMA and Collagen $1 \mathrm{~A} 1$ showed lower fibrosis in ShDR5/GNMT ${ }^{-/-}$mice. (b) Serum transaminases and bilirubin were lower in ShDR5/GNMT ${ }^{-1-}$ mice compared with $\mathrm{GNMT}^{-/-}$mice after BDL. (c) H\&E staining (left panels) and (d) further quantification (upper panel), (c) TUNEL assay (right panels), and (d) quantification of caspase-3 activity (lower panel) showed an obvious attenuation of both necrotic and apoptotic cell death in ShDR5/GNMT ${ }^{-1-}$ mice. (e) Kaplan-Meier curve showing increased survival of ShDR5/GNMT ${ }^{-1-}$ mice compared with GNMT $^{-1-}$ littermates. $n=5-7 .{ }^{*} P<0.05 ;{ }^{* *} P<0.01 ;{ }^{* * *} P<0.001$ (GNMT $^{-1-}$ vs ShDR5/GNMT ${ }^{-1-}$ ). Error bars represent s.d.

further supporting the increased incidence of BDL-induced necrosis. Accordingly, TRAIL ${ }^{-1-} / \mathrm{GNMT}^{-1-}$ hepatocytes were susceptible to necrotic rather than to apoptotic cell death in response to BA. Furthermore, our present data support the selective activation of HSC through phagocytosis of apoptotic hepatocytes (reviewed in Mehal and Imaeda ${ }^{29}$ ), as fibrogenesis was impaired in $\mathrm{TRAIL}^{-1-} / \mathrm{GNMT}^{-1-}$ mice despite severe necrotic cell death and the potential prosurvival environment for HSC in a TRAIL-free context. Interestingly, protection against BDL-induced liver injury 
and fibrogenesis in $\mathrm{GNMT}^{-1-}$ mice after depletion of NK cells and in TRAIL ${ }^{-1-} / \mathrm{GNMT}^{-1-}$ mice all correlated with downregulation of DR5. These data support previous work highlighting the feedback regulation of ligand and receptor. ${ }^{30}$ Finally, in vivo DR5 silencing protected the GNMT-deficient liver against $\mathrm{BDL}$ injury, pointing to DR5 as a key mediator of fibrogenesis by means of hepatocyte death and further HSC activation, rather than acting as an antifibrotic agent causing HSC apoptosis. This antifibrogenic effect likely occurs in the pathological context of GNMT deficiency as, accordingly with the antifibrogenic role of DR5 as a mediator of HSC death, in vivo silencing of DR5 in WT animals led to increased fibrogenesis after BDL in these healthy animals (data not shown).

Cell death triggers the inflammatory response in the liver, which is followed by tissue remodeling and fibrogenesis. Previous work highlighted the implication of KCs during fibrogenesis in different experimental models. ${ }^{20} \mathrm{KCs}$ are recruited to the sites of inflammation and contribute to HSC activation, promoting liver fibrosis as part of the wound healing and regeneration process after injury. ${ }^{21}$ Interestingly, despite increased fibrosis found in the context of GNMT deficiency, KCs were poorly activated after BDL suggesting a negative regulatory feedback with NK cells. Accordingly, depletion of NK cells partially restored the activation of KC, which was, nevertheless, insufficient to trigger fibrogenesis in $\mathrm{GNMT}^{-1-}$ livers likely due to the (transient) absence of TRAIL-cell death. The higher presence of KC in the liver of WT animals that was stimulated with poly I:C (which activates NK cells) would suggest that the attenuation of $\mathrm{KC}$ activation in the presence of activated NK cells is restricted to the context of GNMT absence (data not shown). Overall, it is tempting to speculate that due to the absence of KC activation found in $\mathrm{GNMT}^{-/-}$mice, removal of apoptotic cell debris by HSC may further contribute to their activation and fibrogenic activity. The increased expression of TLR9 consistently observed in $\mathrm{GNMT}^{-1-}$ mice supports this mechanism, as DNA from mammalian apoptotic cells promotes phagocytosis in HSC, ${ }^{7}$ which readily become activated and resistant to TRAIL/DR5-mediated death. ${ }^{26}$

In summary, we here describe that NK cells mediate severe liver injury, further leading to fibrosis through the TRAIL/ DR5 axis in the context of absence of GNMT. We propose that this undergoes through (i) NK cell-mediated direct apoptotic killing of GNMT-deficient hepatocytes by TRAIL, which further triggers an inflammatory response that (ii) activates HSC-mediated fibrogenesis. In accordance, we here show that both NK cell inhibition and overall depletion of TRAIL significantly attenuates liver injury after BDL and further HSC activation and consequent fibrogenesis in $\mathrm{GNMT}^{-/-}$mice. Ultimately, silencing of DR5 also protected the liver against hepatocyte cell death and further fibrogenesis in the absence of GNMT. These data are of relevance as GNMT deficiency occurs in chronic human liver disease regardless of the etiology. Moreover, we here show that even at early stages of human liver (cholestatic) disease, when fibrosis is still not prominent, GNMT expression is low. These data, together with previous work showing attenuation of GNMT expression during NAFLD, ${ }^{24}$ support the essential role of GNMT in maintaining liver homeostasis and preventing liver disease.

Overall, our present study provides new insights on the implication of TRAIL-producing NK cells in mediating liver injury and fibrogenesis when GNMT is absent. This knowledge may facilitate the development of potential therapeutic strategies based on NK cell inactivation to counteract hepatocyte injury in the pathogenic context of GNMT deficiency, a scenario that is critical for the progression from chronic to end-stage liver disease. Finally, our work underlines the importance of carefully assessing the molecular context of liver disease before proposing therapeutic strategies to counteract fibrogenesis in cirrhotic patients from different etiologies.

Supplementary Information accompanies the paper on the Laboratory Investigation website (http://www.laboratoryinvestigation.org)

\section{ACKNOWLEDGMENTS}

This work was supported by grants from the Instituto de Salud Carlos III; FIS, PS12/00402 (to NB), NIH AT-1576 (to SCL, MLM-C, and JMM), ETORTEK-2012 (to MLM-C), Sanidad del Gobierno Vasco 2013 (to MLM-C), Pl11/01588 (to MLM-C). Plan Nacional SAF2011-29851 (to JMM). NB is funded by the

Program Ramon y Cajal (Ministry of Economy and Competitiveness, Spain). PI11/02942 (to AL). Ciberehd is funded by the Instituto de Salud Carlos III.

\section{DISCLOSURE/CONFLICT OF INTEREST}

The authors declare no conflict of interest.

1. Martinez-Lopez N, Varela-Rey M, Ariz U, et al. S-adenosylmethionine and proliferation: new pathways, new targets. Biochem Soc Trans 2008;36:848-852.

2. Avila MA, Berasain C, Torres $L$, et al. Reduced mRNA abundance of the main enzymes involved in methionine metabolism in human liver cirrhosis and hepatocellular carcinoma. J Hepatol 2000;33:907-914.

3. Martinez-Chantar ML, Vazquez-Chantada M, Ariz U, et al. Loss of the glycine $\mathrm{N}$-methyltransferase gene leads to steatosis and hepatocellular carcinoma in mice. Hepatology 2008;47:1191-1199.

4. Gomez-Santos L, Luka Z, Wagner C, et al. Inhibition of natural killer cells protects the liver against acute injury in the absence of glycine N-methyltransferase. Hepatology 2012;56:747-759.

5. Bataller R, Brenner DA. Liver fibrosis. J Clin Invest 2005;115:209-218.

6. Canbay A, Friedman S, Gores GJ. Apoptosis: the nexus of liver injury and fibrosis. Hepatology 2004;39:273-278.

7. Watanabe A, Hashmi A, Gomes DA, et al. Apoptotic hepatocyte DNA inhibits hepatic stellate cell chemotaxis via toll-like receptor 9. Hepatology 2007;46:1509-1518.

8. Syn WK, Agboola KM, Swiderska M, et al. NKT-associated hedgehog and osteopontin drive fibrogenesis in non-alcoholic fatty liver disease. Gut 2012;61:1323-1329.

9. Radaeva S, Wang L, Radaev S, et al. Retinoic acid signaling sensitizes hepatic stellate cells to NK cell killing via upregulation of NK cell activating ligand RAE1. Am J Physiol Gastrointest Liver Physiol 2007;293:G809-G816.

10. Radaeva S, Sun R, Jaruga B, et al. Natural killer cells ameliorate liver fibrosis by killing activated stellate cells in NKG2D-dependent and 
tumor necrosis factor-related apoptosis-inducing ligand-dependent manners. Gastroenterology 2006;130:435-452.

11. Shimoda $\mathrm{S}$, Harada $\mathrm{K}$, Niiro $\mathrm{H}$, et al. Interaction between Toll-like receptors and natural killer cells in the destruction of bile ducts in primary biliary cirrhosis. Hepatology 2011;53:1270-1281.

12. Chuang $\mathrm{YH}$, Lian ZX, Tsuneyama $\mathrm{K}$, et al. Increased killing activity and decreased cytokine production in NK cells in patients with primary biliary cirrhosis. J Autoimmun 2006;26:232-240.

13. Takeda K, Kojima Y, Ikejima K, et al. Death receptor 5 mediatedapoptosis contributes to cholestatic liver disease. Proc Natl Acad Sci USA 2008;105:10895-10900.

14. Kahraman A, Barreyro FJ, Bronk SF, et al. TRAIL mediates liver injury by the innate immune system in the bile duct-ligated mouse. Hepatology 2008:47:1317-1330.

15. Kahraman A, Schlattjan M, Kocabayoglu P, et al. Major histocompatibility complex class I-related chains $A$ and $B(\mathrm{MIC} \mathrm{A/B):} \mathrm{a} \mathrm{novel} \mathrm{role} \mathrm{in}$ nonalcoholic steatohepatitis. Hepatology 2010;51:92-102.

16. Luka Z, Capdevila A, Mato JM, et al. A glycine N-methyltransferase knockout mouse model for humans with deficiency of this enzyme. Transgenic Res 2006;15:393-397.

17. Fickert $\mathrm{P}$, Zollner $\mathrm{G}$, Fuchsbichler $\mathrm{A}$, et al. Ursodeoxycholic acid aggravates bile infarcts in bile duct-ligated and Mdr2 knockout mice via disruption of cholangioles. Gastroenterology 2002;123:1238-1251.

18. Beraza N, Ludde T, Assmus U, et al. Hepatocyte-specific IKK gamma/ NEMO expression determines the degree of liver injury. Gastroenterology 2007;132:2504-2517.

19. Beraza N, Malato $\mathrm{Y}$, Sander LE, et al. Hepatocyte-specific NEMO deletion promotes NK/NKT cell- and TRAIL-dependent liver damage. J Exp Med 2009;206:1727-1737.

20. Seki E, De Minicis S, Osterreicher $\mathrm{CH}$, et al. TLR4 enhances TGF-beta signaling and hepatic fibrosis. Nat Med 2007:13:1324-1332.
21. Seki E, De Minicis S, Gwak GY, et al. CCR1 and CCR5 promote hepatic fibrosis in mice. J Clin Invest 2009;119:1858-1870.

22. Smyth MJ, Cretney E, Takeda K, et al. Tumor necrosis factor-related apoptosis-inducing ligand (TRAIL) contributes to interferon gammadependent natural killer cell protection from tumor metastasis. J Exp Med 2001;193:661-670.

23. Takeda K, Hayakawa Y, Smyth MJ, et al. Involvement of tumo necrosis factor-related apoptosis-inducing ligand in surveillance of tumor metastasis by liver natural killer cells. Nat Med 2001;7: 94-100.

24. Chen $\mathrm{CH}$, Huang $\mathrm{MH}$, Yang JC, et al. Prevalence and risk factors of nonalcoholic fatty liver disease in an adult population of taiwan: metabolic significance of nonalcoholic fatty liver disease in nonobese adults. J Clin Gastroenterol 2006;40:745-752.

25. Gao B, Radaeva S. Natural killer and natural killer T cells in liver fibrosis Biochim Biophys Acta 2013;1832:1061-1069.

26. Jiang JX, Mikami K, Venugopal S, et al. Apoptotic body engulfment by hepatic stellate cells promotes their survival by the JAK/STAT and Akt/NF-kappaB-dependent pathways. J Hepatol 2009;51: 139-148.

27. Liedtke C, Bangen JM, Freimuth J, et al. Loss of caspase-8 protects mice against inflammation-related hepatocarcinogenesis but induces non-apoptotic liver injury. Gastroenterology 2011;141:2176-2187.

28. Galluzzi L, Kepp O, Kroemer G. FADD: an endogenous inhibitor of RIP3-driven regulated necrosis. Cell Res 2011;21:1383-1385.

29. Mehal W, Imaeda A. Cell death and fibrogenesis. Semin. Liver Dis 2010;30:226-231.

30. Shetty S, Gladden JB, Henson ES, et al. Tumor necrosis factor-related apoptosis inducing ligand (TRAIL) up-regulates death receptor 5 (DR5) mediated by NFkappaB activation in epithelial derived cell lines. Apoptosis 2002;7:413-420. 